\title{
Power-Dependent Polarization Switching and Pulse Narrowing in a Semiconductor Quantum Well Amplifier
}

\author{
Ming-Shan Lin, Din-Wei Huang, and C. C. Yang \\ Institute of Electro-Optical Engineering and \\ Department of Electrical Engineering, \\ National Taiwan University \\ Taipei, Taiwan, R.O.C.
}

\begin{abstract}
Gain/absorption saturation in semiconductors is a widely used nonlinear optical mechanism for all-optical ultrafast switching, such as the devices based on nonlinear directional coupling. In quantum well structures, because of different selection rules for electronic transitions between the TE and TM modes, the two polarization modes manifest quite different linear and nonlinear optical properties.
\end{abstract} The nonlinear anisotropy can lead to power-dependent polarization evolution when an optical signal propagates through such a device.

In this paper, we report the experimental results of efficient nonlinear polarization switching in a single quantum well amplifier and show that the numerical simulations based on a simple model agree reasonably well with the experimental data. The sample consisted of an InGaAs well, GaAs barriers, and graded AlGaAs cladding layers. It lased at $974 \mathrm{~nm}$. The electro-luminescence shows a peak near $970 \mathrm{~nm}$ in the TE mode and a peak near $940 \mathrm{~nm}$ in the TM mode, roughly indicating the spectral positions of the heavy- and light-hole transitions. Hence, for signal wavelengths longer than $940 \mathrm{~nm}$ the TM mode is passive or transparent although the TE mode may experience gain or absorption, depending on injection current level and wavelength. Near or below $940 \mathrm{~nm}$, both TE and TM modes become active.

We used a Ti:Sapphire laser, pumped by an Argon laser, to provide cw and $2-5$ ps signals. Figure 1 shows the output polarizations of a $\mathrm{cw}$ and a ps signal at $940 \mathrm{~nm}$ with the same input average power at $70 \mathrm{~mW}$. With the same input linear polarization ( 45 degrees) at zero injection current, the output polarizations are quite different due to different absorption saturation. The marks in Fig. 2 shows the switching ratios of both $\mathrm{cw}$ and ps signals with the horizontal axis representing the input average power coupled into the waveguide. The signal wavelength is 940 $\mathrm{nm}$, the cw injection current is $20 \mathrm{~mA}$, and the input linear polarization is 15 degrees from the TE direction. Although the $\mathrm{cw}$ signal does not experience any switching effect, the ps signal does show efficient power-dependent polarization switching. Figure 3 demonstrates the absolute output powers for the ps signal case. From the 
trends of the curves, it is clear that in this situation the TE mode experiences absorption saturation and the TM mode experiences gain saturation. In numerical simulations, we solve the rate equations of the carrier density and the optical wave fields in the two modes. The results corresponding to the experimental data in Figs. 2 and 3 are also shown with the continuous curves in these two figures, indicating reasonable agreement.

Because the polarization evolution in such a device is power-dependent, it is expected that in a well-chosen output polarization direction, the pulsed signals can be narrowed. The numerical simulation results of repeated propagation of a pulse with pulsewidth 0.1 times the carrier lifetime through such an amplifier are shown in Fig. 4. Pulse narrowing can be observed.

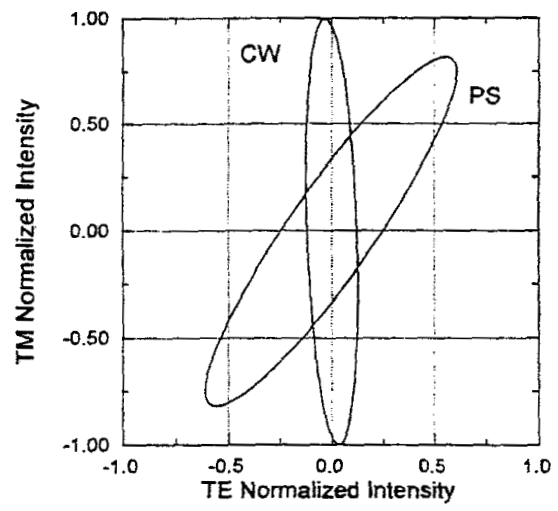

Fig. 1 Comparison of output polarizations of $\mathrm{cW}$ and ps signals.

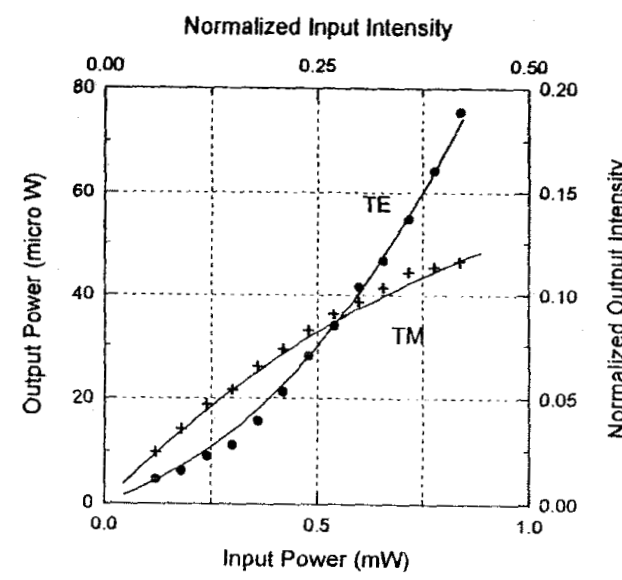

Fig. 3 Output power/intensity with ps in the case of Fig. 2.

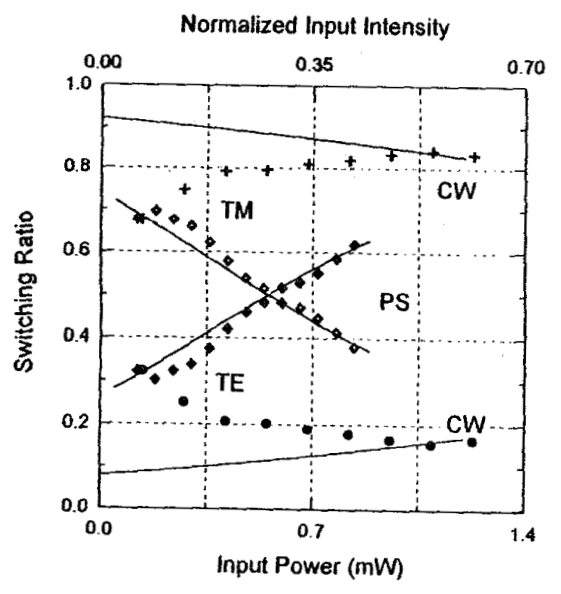

Fig. 2 Switching ratio at $940 \mathrm{~nm}$.

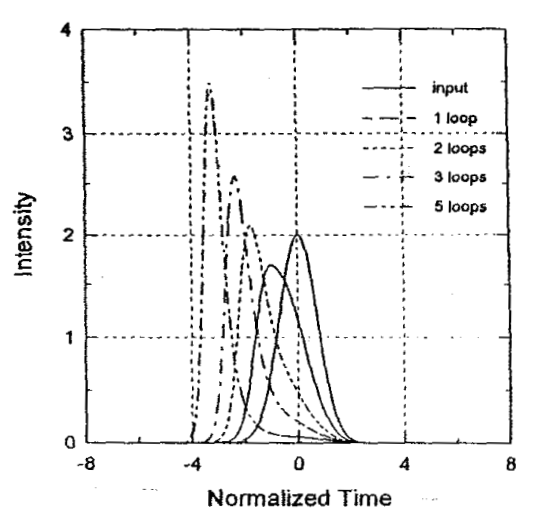

Fig. 4 Simulation of pulse narrowing. 